\title{
Optimal Control of a Grid-Connected Hybrid Electrical Energy Storage System for Homes
}

\author{
Yanzhi Wang, Xue Lin, and Massoud Pedram \\ University of Southern California \\ Los Angeles, CA, USA 90089 \\ \{yanzhiwa, xuelin, pedram\}@usc.edu
}

\author{
Sangyoung Park and Naehyuck Chang \\ Seoul National University \\ Seoul, Korea 151-744 \\ \{sypark, naehyuck\}@elpl.snu.ac.kr
}

\begin{abstract}
Integrating residential photovoltaic (PV) power generation and electrical energy storage (EES) systems into the Smart Grid is an effective way of utilizing renewable power and reducing the consumption of fossil fuels. This has become a particularly interesting problem with the introduction of dynamic electricity energy pricing models since electricity consumers can use their PV-based energy generation and EES systems for peak shaving on their power demand profile from the grid, and thereby, minimize their electricity bill. Due to the characteristics of a realistic electricity price function and the energy storage capacity limitation, the control algorithm for a residential EES system should accurately account for various energy loss components during operation. Hybrid electrical energy storage (HEES) systems are proposed to exploit the strengths of each type of EES element and hide its weaknesses so as to achieve a combination of performance metrics that is superior to those of any of its individual EES components. This paper introduces the problem of how best to utilize a HEES system for a residential Smart Grid user equipped with PV power generation facilities. The optimal control algorithm for the HEES system is developed, which aims at minimization of the total electricity cost over a billing period under a general electricity energy price function. The proposed algorithm is based on dynamic programming and has polynomial time complexity. Experimental results demonstrate that the proposed HEES system and optimal control algorithm achieves $73.9 \%$ average profit enhancement over baseline homogeneous EES systems.
\end{abstract}

Keywords-hybrid electrical energy storage system; smart grid; optimal control

\section{INTRODUCTION}

The traditional (static and centrally controlled) structure of the national electricity grid (a.k.a. the Power Grid) consists of a transmission network, which transmits electrical power generated at remote power plants through long-distance high-voltage power lines to substations, and a distribution network, which delivers electrical power from substations to local end users. In this infrastructure, the local distribution network is often statically adjusted to match the load profile from its end users. The Power Grid must be able to support the worst-case power demand of all end users in order to avoid potential power delivery failure as the end user profiles often change drastically according to the day of week and time of day [1].

Progressive integration of smart meters and communication devices aims to transform the Power Grid to a decentralized Smart Grid, which can monitor and control the power flow in the Grid to match the amount of power generation to that of power consumption, and to minimize the overall cost of electrical energy delivered to the end users [2]. The Smart Grid approach avoids expending a large amount of capital in the future for expanding the power generation

This work is supported in part by a grant from the U.S. National Science Foundation, and by a grant from the BK21 Project and the NRF of Korea funded by the MEST (No. 20120005640). capacity to meet the expected growth of energy consumption among end users under the worst-case power demand conditions. In the Smart Grid infrastructure, utility companies can employ dynamic electricity pricing strategies incentivizing consumers to perform demand side management by adjusting their power demand from the Grid to match the power generation capacity of the Grid. There are several ways to perform such a demand side management [3]. In this paper, we focus on integrating PV power generation with the Smart Grid for residential usage.

Integrating residential-level renewable energy sources into the Smart Grid is an effective way for utilizing renewable power and reducing the consumption of fossil fuels. However, we must address one important problem for these benefits to be realized. There exists a mismatch between the peak PV power generation time (usually at noon) and the peak load power consumption time (usually in the evening) in each day. This timing skew results in conditions where the generated PV power cannot be optimally utilized to perform peak load power shaving. Electrical energy storage (EES) systems are thus deployed for residential users to mitigate the supply-demand mismatch, increase power availability and regulate the peak-power demand of load devices [4], [5], [6], [16].

The proposed residential-level EES system stores power from the Smart Grid during off-peak periods of each day and from the PV system, and provides power for the end users during the peak periods of that day for peak power shaving and energy cost reduction (since electrical energy tends to be more expensive during these peak hours.) The design of energy pricing-aware control algorithm for the residential-level EES system, which controls the charging and discharging of the EES system and magnitude of charging/discharging current, is an important task in order for the Smart Grid technology to deliver on its promises [16]. Residentiallevel EES systems are the key for shortening the long break-even time and popularizing the renewable energy source installations.

State-of-the-art EES system deployments are mainly homogeneous [6], i.e., they consist of a single type of EES element. However, none of the existing EES elements can simultaneously fulfill all the desired features of an ideal EES system, to name a few, high charge/discharge efficiency, high energy density, low cost per unit capacity, and long cycle life. Hence, the overall performance of a homogeneous EES system is limited by the underlying EES element characteristics, thereby restricting the wide application of EES systems for household uses.

In order to overcome these limitations, a novel energy storage technology, the hybrid EES (HEES) system, is currently gaining popularity [7], [8]. A HEES system comprises of several heterogeneous EES elements and is therefore able to exploit the strengths of each type of EES element while hiding its weaknesses, so as to achieve a combination of performance metrics that is superior to those of any of its individual EES components. The HEES system is also beneficial in terms of capital cost compared with the homogenous EES system with the same performance. Design 
considerations and control policies of the HEES systems have been proposed [9], [10], [11] to help realize the potential benefits. Despite these research efforts, the lack of investigation on the HEES system applications, such as integrating residential-level HEES systems in the Smart Grid, significantly restrains its wide adoption. Appropriate control algorithm for a residential-level HEES system will be more complicated than the control algorithm for a homogeneous EES system [16] since the former requires taking into account the distinct and complementary characteristics of various types of EES elements.

This paper is the first to formally describe the integration of a residential-level HEES system for the Smart Grid users equipped with PV power generation. This paper is the first to propose the optimal control algorithm for the HEES system. The objective of the optimal control algorithm is to reduce the total electricity cost over a billing period and perform peak power shaving under arbitrary energy price function set by the Smart Grid central controller. The proposed optimal HEES system control algorithm correctly accounts for the distinct characteristics of different types of EES elements, conversion efficiency variations of power converters, as well as the time-of-use (TOU) dependent energy price function. The proposed control algorithm is based on dynamic programming and therefore has polynomial time complexity. Experimental results demonstrate that the proposed HEES system and control algorithm achieves $73.9 \%$ average profit enhancement over homogeneous EES systems.

\section{HEES SYSTEMS}

\section{A. HEES System Architecture and Control}

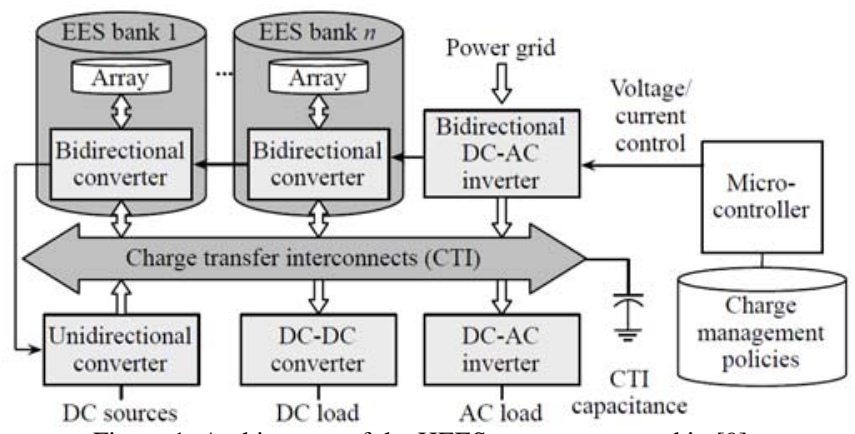

Figure 1: Architecture of the HEES system proposed in [9].

Figure 1 illustrates the HEES system architecture proposed in [9]. The system comprises multiple heterogeneous EES banks, connected with each other through the Charge Transfer Interconnect (CTI). Each EES bank consists of an EES (element) array and a bidirectional converter. The EES array is composed of multiple homogeneous EES elements with the same state-of-charge (SoC), organized in a two-dimensional array using series and/or parallel connections. The bidirectional converters control power transfer into and out of the EES array through the CTI regardless of their different voltages. The bidirectional converter is typically implemented based on a switching-mode power converter and can be configured as either a voltage regulator or a current regulator:

- Voltage regulating mode: The converter generates a controllable voltage output regardless of input and output current variation.

- Current regulating mode: The converter generates a controllable current output regardless of input and output voltage variation.

The HEES system has unidirectional converters for the DC power source and DC load. We also use a bidirectional DC-AC inverter connecting to the Power Grid. They have the same regulating modes as the bidirectional converters in the EES banks.

The HEES system uses a microcontroller as the main controller to determine the operation of the converters [9]. It determines the voltage level of the CTI, the amount of charging/discharging current of each EES bank, and current drawn from the power source according to the charge management policies. This method enables us to control both the CTI voltage and the EES bank current. We set only one converter in the voltage regulating mode and let it control the CTI voltage. All others operate in the current regulating mode. The output current of the voltage regulating converter is automatically determined so that the sum of input currents of the CTI is equal to the sum of output currents. This control method makes the feedback control loop in the converters maintain the stability of their output voltage/current while the software real-time control tasks in the microcontroller provide high-level charge management policies.

\section{B. EES Element Array Model}

The most commonly used EES elements are lead-acid batteries, Lithium-ion (Li-ion) batteries, and supercapacitors. Due to supercapacitor's high capital cost [7], household EES systems typically consist of lead-acid batteries or Li-ion batteries. In this paper, we present the optimal residential-level HEES controlling algorithm in the Smart Grid with these two representative EES elements. Generally speaking, lead-acid batteries are much cheaper than Li-ion batteries, yet they suffer from a shorter cycle life and incur higher power loss during charging and discharging due to their larger internal resistance and more severe rate capacity effect.

The voltaic representation of the EES array SoC is given by:

$$
\boldsymbol{V}_{\text {soc }}=C_{\text {array }} / C_{\text {full }} \times 1 \mathrm{~V},
$$

where $C_{\text {array }}$ is the amount of charge stored in the EES array, and $C_{\text {full }}$ is the amount of charge in the EES array when it is fully charged. We derive $C_{\text {full }}$ in Coulomb from the EES array capacity Capacity given in Ahr:

$$
C_{\text {full }}=3600 \times \text { Capacity. }
$$

We interpret $\boldsymbol{V}_{\boldsymbol{S O} \boldsymbol{C}}$ as the state of the EES array.

EES array models specify the relationship among $\boldsymbol{V}_{\boldsymbol{S O} \boldsymbol{C}}$, the array open circuit voltage (OCV) $V_{\text {array }}^{O C}$, closed circuit voltage (CCV) $V_{\text {array }}^{C C}$, array stored energy $E_{\text {array }}$, and array current $I_{\text {array }}$. We use an electronic equivalent circuit model in [12] as the battery model, which is suitable for developing the mathematical formulation. As shown in Figure 2, it includes a runtime-based model on the left as well as a circuit-based model on the right for accurate capturing of the battery array service life and I-V characteristics. In Figure 2, $R_{s}$, $R_{t s}$ and $R_{t l}$ are internal resistances, and $C_{t s}, C_{t l}$ are internal capacitances, of the battery array. We introduce details of the EES array model in the following three aspects: OCV-SoC relationship, CCV-OCV relationship, and rate capacity effect.

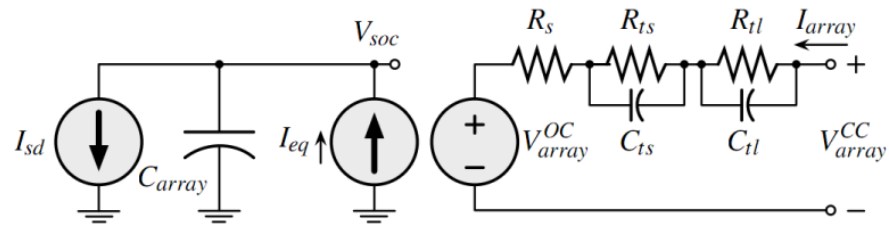

Figure 2: Battery array equivalent circuit model.

\section{1) $\mathrm{OCV}$-SoC Relationship:}

$V_{\text {array }}^{O C}$ is a monotonically increasing function of $\boldsymbol{V}_{\text {SoC }} \cdot V_{\text {array }}^{O C}$ is modeled as a voltage-controlled voltage source controlled by $\boldsymbol{V}_{\boldsymbol{S O C}}$ of the battery array as shown in Figure 2. The OCV-SoC relationship is nonlinear and is given by

$$
V_{\text {array }}^{O C}=b_{1} e^{b_{2} V_{S o c}}+b_{3} \boldsymbol{V}_{S O c^{3}}+b_{4} \boldsymbol{V}_{S O c^{2}}{ }^{2}+b_{5} \boldsymbol{V}_{S O C}+b_{6},
$$


where those $b_{i}$ 's are empirically determined parameters from real pulsed charging and discharging measurements [13].

\section{2) CCV-OCV Relationship:}

$V_{\text {array }}^{C C} \neq V_{\text {array }}^{O C}$ when $I_{\text {array }} \neq 0$ for the battery array due to internal resistances and capacitances. The relationship between $V_{\text {array }}^{C C}$ and $V_{\text {array }}^{O C}$ of the battery array is given by

$$
V_{\text {array }}^{C C}=V_{\text {array }}^{O C}+V_{t l}+V_{t s}+I_{\text {array }} \cdot R_{s},
$$

where $V_{t l}$ and $V_{t s}$ are the voltage drops across the internal capacitances [12]. Generally speaking, lead-acid batteries have larger internal resistances and capacitances than Li-ion batteries.

\section{3) Rate Capacity Effect and Coulomb Counting:}

The rate capacity effect of batteries explains that the charging and discharging efficiencies decrease with the increasing of charging and discharging currents, respectively. More precisely, the Peukert's formula [14] describes that the charging and discharging efficiencies of a battery element array, as functions of the charging current $I_{c}$ and discharging current $I_{d}$, respectively, are given by

$$
\eta_{\text {rate }, c}\left(I_{c}\right)=\frac{k_{c}}{\left(I_{c}\right)^{\alpha_{c}}}, \quad \eta_{\text {rate }, d}\left(I_{d}\right)=\frac{k_{d}}{\left(I_{d}\right)^{\alpha_{d}}},
$$

where $k_{c}, \alpha_{c}, k_{d}$, and $\alpha_{d}$ are constants known a priori. Lead-acid batteries are subject to a much more severe rate capacity effect compared with Li-ion batteries. We define the equivalent current inside the battery array as the actual charge accumulating/reducing speed inside the battery array, given by:

$$
I_{e q}=\left\{\begin{array}{cc}
I_{\text {array }} \cdot \eta_{\text {rate }, c}\left(I_{\text {array }}\right), & \text { if } I_{\text {array }}>0, \\
I_{\text {array }} / \eta_{\text {rate }, d}\left(\left|I_{\text {array }}\right|\right), & \text { if } I_{\text {array }}<0 .
\end{array}\right.
$$

\section{Converter and Inverter Model}

We use a PWM (pulse width modulation) buck-boost switching converter as the (bidirectional) converter in the HEES system to accommodate a wide range of EES array terminal voltages. The converter power consumption model is provided in [9]. Generally speaking, the power conversion efficiency of a converter is a function of its input and output voltages and currents. The conversion efficiency is maximized when the converter output current is within a small operation range, and its input and output voltages are close to each other. DC-AC inverters have similar properties, with power consumption model provided in [16].

\section{PROBLEM Formulation}

Figure 3 presents the architecture of the proposed HEES system integrated for a residential Smart Grid user with PV power generation utility. Similar system architecture has been investigated in [16]. However, the major difference is that we incorporate a HEES system for the residential-level Smart Grid user while [16] only considers a homogenous EES system.

We use a slotted time model, i.e., all the system constraints as well as decisions are provided for discrete time intervals of equal length. More specifically, each day is divided into $N$ time slots, each of duration $\Delta_{T}$. We consider the residential electric load with load current $I_{\text {load }}[i]$ at the $i$-th time slot. We consider the HEES system consisting of a lead-acid battery bank and a Li-ion battery bank in this paper. Our HEES system control algorithm is able to handle more complicated HEES systems with relatively high computation complexity. The proposed optimal HEES system control algorithm is performed at the beginning of a day and it optimally determines the CTI voltage level $V_{C T I}[i](1 \leq i \leq N)$ and charging or discharging current for each EES array throughout the day. The other voltage or current values are either given or associate variables. The proposed algorithm should correctly account for the distinct characteristics of different types of EES elements, conversion efficiency variation of power converters, and the TOU dependent energy price function.

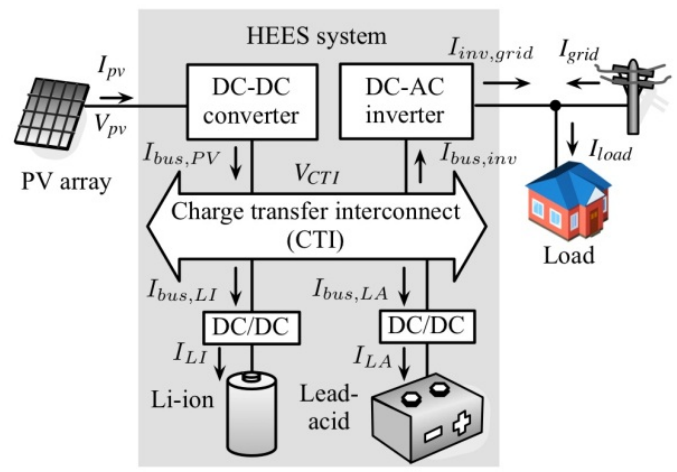

Figure 3: Architecture of the proposed HEES system for a residential Smart Grid user with PV power generation.

Let $I_{L A}[i]$ and $I_{b u s, L A}[i]$ denote the charging/discharging current of the lead-acid battery array and bank at the $i$-th time slot, respectively. $I_{L A}[i]$ and $I_{b u s, L A}[i]$ are positive when charging the battery array and negative when discharging the battery array. We denote the equivalent current inside the lead-acid battery array by $I_{e q, L A}[i]$. The relationship between $I_{e q, L A}[i]$ and $I_{L A}[i]$ are given in Section II.B. We denote the SoC of the lead-acid battery array by $V_{S O C, L A}[i]$, which is calculated from its initial SoC, $V_{S O C, L A}[1]$, using Coulomb counting:

$$
V_{S O C, L A}[i]=V_{S O C, L A}[1]+\frac{\sum_{j=1}^{i-1} I_{e q, L A}[j] \cdot \Delta_{T}}{C_{f u l l, L A}} .
$$

We denote the OCV and CCV of the lead-acid battery array by $V_{\text {array }, L A}^{O C}[i]$ and $V_{\text {array, } L A}^{C C}[i]$, respectively. The OCV-SoC relationship and CCV-OCV relationship are given in Section II.B. Similar notations also apply for the Li-ion battery bank by replacing the subscript $L A$ by $L I$.

We apply the maximum power point tracking (MPPT) method for the PV panel, which ensures that the output power of the PV panel is maximized [15]. The PV panel output voltage and current are denoted by $V_{P V}[i]$ and $I_{P V}[i]$, respectively, and they are given to the HEES system controller. The grid voltage $V_{\text {grid }}$ is a constant value (say, 110 V.) We denote the current flowing from the DC-AC inverter to the Grid by $I_{\text {inv,grid }}[i] . I_{\text {inv, grid }}[i]$ can be positive (i.e., power flows from the HEES system to the AC load) or negative (i.e., power flows from the Grid to the HEES system.) The current drawn from the Grid, denoted by $I_{\text {grid }}[i]$, satisfies $I_{\text {grid }}[i]=I_{\text {load }}[i]-I_{\text {inv,grid }}[i]$. We assume that $I_{\text {grid }}[i] \geq 0$. In other words, the Smart Grid user cannot get reimbursed from selling power back into the Grid.

The power conversion circuitries exploited in the system accounts for a significant portion of power losses. We denote the power losses of various converters and the DC-AC inverter at the $i$-th time slot by $P_{c o n v, L A}[i], P_{c o n v, L I}[i], P_{c o n v, P V}[i]$, and $P_{\text {inv }}[i]$, which are functions of their input and output voltages and currents [9], [16]. We have the following equations due to the energy conservation law:

$$
\begin{gathered}
V_{P V}[i] \cdot I_{P V}[i]=P_{c o n v, P V}[i]+V_{C T I}[i] \cdot I_{b u s, P V}[i], \\
V_{C T I}[i] \cdot I_{b u s, L A}[i]=P_{c o n v, L A}[i]+V_{a r r a y, L A}^{C C}[i] \cdot I_{L A}[i], \\
V_{C T I}[i] \cdot I_{b u s, L I}[i]=P_{c o n v, L I}[i]+V_{\text {array }, L I}^{C C}[i] \cdot I_{L I}[i], \\
V_{C T I}[i] \cdot I_{\text {bus }, \text { inv }}[i]=P_{\text {inv }}[i]+V_{\text {grid }} \cdot I_{\text {inv,grid }}[i],
\end{gathered}
$$




$$
I_{b u s, P V}[i]=I_{b u s, L A}[i]+I_{b u s, L I}[i]+I_{b u s, i n v}[i] .
$$

Equations (9) - (11) also hold when the corresponding currents are negative (i.e., power flows in the opposite direction.)

The objective of the HEES system control algorithm is to maximize the total energy cost saving, or equivalently, minimizing the total energy cost, over a billing period (i.e., one day.) The electricity energy price function is pre-announced by the utility company just before the start of each billing period, and the price function does not change until the start of the next billing period. Let Price $[i]$ denote the arbitrary unit energy price at the $i$-th time slot. The optimal HEES system control problem is formally described as follows:

Given: $\mathrm{PV}$ panel output profile $V_{P V}[i], I_{P V}[i]$, residential load demand profile $V_{\text {grid }}, I_{\text {load }}[i]$, and the TOU dependent energy price Price $[i]$ for $1 \leq i \leq N$, initial SoCs of EES arrays $V_{S O C, L A}[1], V_{S O C, L I}[1]$.

Control variables: CTI voltage $V_{C T I}[i]$, battery array charging/discharging current $I_{L A}[i], I_{L I}[i]$ for $1 \leq i \leq N$. The control variables not only determine when to charge or discharge the EES arrays, but also the optimal operating conditions for charging and discharging.

Minimize: the total energy cost for a day, given by

$$
\sum_{i=1}^{N} \text { Price }[i] \cdot V_{\text {grid }} \cdot I_{\text {grid }}[i] \cdot \Delta_{T} \text {. }
$$

\section{Subject to:}

i) Energy Conservation: (8) - (12) are satisfied.

ii) Capacity and Power Rating Constraints: Each EES array SoC cannot be less than zero or more than $100 \%$, i.e.,

$$
0 \leq V_{S O C, L A}[i], V_{S O C, L I}[i] \leq 100 \% .
$$

Moreover, the charging/discharging current of each EES array cannot exceed a maximum value, i.e.,

$$
\begin{gathered}
-I_{L A, M A X, d} \leq I_{L A}[i] \leq I_{L A, M A X, c}, \\
-I_{L I, M A X, d} \leq I_{L I}[i] \leq I_{L I, M A X, c} .
\end{gathered}
$$

iii) Final Stored Energy Constraints: Each EES array SoC at the end of day should be no less than the initial SoC value, i.e.,

$$
V_{S O C, L A}[N+1] \geq V_{S O C, L A}[1], V_{S O C, L I}[N+1] \geq V_{S O C, L I}[1] \text {. }
$$

\section{Optimal HEES System CONTROL AlgORITHM}

We derive the optimal solution of the HEES system control problem based on dynamic programming. This algorithm works for the HEES system and derive the optimal solution while [16] considers the homogeneous EES system only and derive a nearoptimal solution. We consider a variant version of the original HEES system control problem, named the $\left(V_{S O C, 1}, V_{S O C, 2}, i\right)$ problem. This problem minimizes the total electrical energy cost during time slots 1 to $i$ under the condition that $V_{S O C, L A}[i+1]=V_{S O C, 1}$ and $V_{S O C, L I}[i+$ $1]=V_{S O C, 2}$. The following observation describes the relationship between these two optimization problems:

Observation I: Consider the optimal solutions of all the $\left(V_{S O C, 1}, V_{S O C, 2}, i=N\right)$ problems satisfying $V_{S O C, 1} \geq V_{S O C, L A}[1]$ and $V_{S O C, 2} \geq V_{S O C, L I}[1]$ (i.e., satisfying the final stored energy constraints (17)). The optimal solution of the original HEES system control problem is the one that minimizes the total energy cost during the whole day (i.e., over time slots 1 to $N$.)
The optimal substructure property of the $\left(V_{S O C, 1}, V_{S O C, 2}, i\right)$ problem goes as follows. This enables us to apply the dynamic programming method to find the optimal solution of the $\left(V_{S O C, 1}, V_{S O C, 2}, i\right)$ problem efficiently.

Observation II (The Optimal Substructure Property): Suppose that we have found the optimal solution of the $\left(V_{S O C, 1}, V_{S O C, 2}, i\right)$ problem that minimizes the total energy cost during time slots 1 to $i$. Suppose that $V_{S O C, L A}\left[i^{\prime}+1\right]=V_{S O C, 1}^{\prime}$ and $V_{S O C, L I}\left[i^{\prime}+1\right]=V_{S O C, 2}^{\prime}$ $\left(i^{\prime}<i\right)$ in that optimal solution. This corresponds to the subproblem $\left(V_{S O C, 1}^{\prime}, V_{S O C, 2}^{\prime}, i^{\prime}\right)$. The optimal solution of the $\left(V_{S O C, 1}, V_{S O C, 2}, i\right)$ problem contains within it the optimal solution of the subproblem $\left(V_{S O C, 1}^{\prime}, V_{S O C, 2}^{\prime}, i^{\prime}\right)$.

Based on Observations I and II, we introduce Algorithm 1 using the dynamic programming method to find the optimal solution of the original HEES system control problem. We perform Algorithm 1 at the beginning of a day to determine the control variables over all the time slots in that day in a one-shot manner.

\section{Algorithm 1: The optimal solution for the HEES system control problem using dynamic programming}

Input: PV panel output profile $V_{P V}[i], I_{P V}[i]$, load profile $V_{\text {grid }}, I_{\text {load }}[i]$, and energy price Price $[i]$ for $1 \leq i \leq N$, initial array SoCs $V_{S O C, L A}[1], V_{S O C, L I}[1]$

Output: $V_{C T I}[i], I_{L A}[i]$, and $I_{L I}[i]$ for $1 \leq i \leq N$

Initialize Min_Cost $\left(V_{S O C, 1}, V_{S O C, 2}, 0\right)$ in the way as shown in (18)

For $(i=1 ; i \leq N ; i++)$ do:

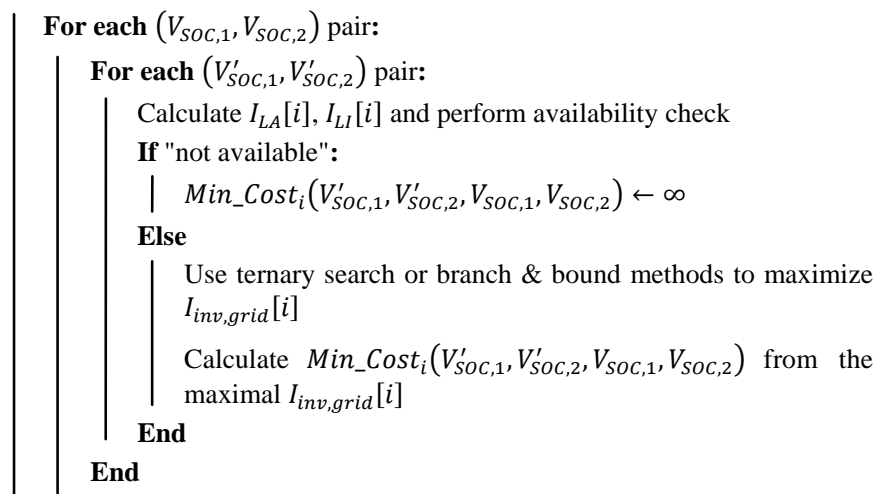

Calculate Min_Cost $\left(V_{S O C, 1}, V_{S O C, 2}, i\right)$, Last_SoC $\operatorname{SA}_{L A}\left(V_{S O C, 1}, V_{S O C, 2}, i\right)$, and Last_SoC LI $_{L}\left(V_{S O C, 1}, V_{S O C, 2}, i\right)$ using (20) and (21)

End

End

Determine $V_{S O C, L A}[N+1]$ and $V_{S O C, L I}[N+1]$ using (22)

Perform tracing back using the Min_Cost, Last_SoC $C_{L A}$, and Last_SoC matrices, as well as the above-obtained $\mathrm{SoC}$ values at the end of day, to determine the optimal $V_{C T I}[i], I_{L A}[i]$, and $I_{L I}[i]$ for $1 \leq i \leq N$

Return the optimal $V_{C T I}[i], I_{L A}[i]$, and $I_{L I}[i]$ for $1 \leq i \leq N$

For $\quad 0 \leq V_{S O C, 1} \leq 100 \% \quad$ and $\quad 0 \leq V_{S O C, 2} \leq 100 \%$, $\operatorname{Min} \_\operatorname{Cost}\left(V_{S O C, 1}, V_{S O C, 2}, i\right)$ denotes the minimal amount of electricity energy cost during time slots 1 to $i$ under the condition that $V_{S O C, L A}[i+1]=V_{S O C, 1}$ and $V_{S O C, L I}[i+1]=V_{S O C, 2}$. We initialize the matrix Min_Cost as:

$\operatorname{Min} \_\operatorname{Cost}\left(V_{S O C, 1}, V_{S O C, 2}, 0\right)=$

$\left\{\begin{array}{l}0, \text { if } V_{S O C, 1}=V_{S O C, L A}[1] \text { and } V_{S O C, 2}=V_{S O C, L I}[1], \\ \infty, \quad \text { otherwise. }\end{array}\right.$ 
Algorithm 1 calculates $\operatorname{Min} \_\operatorname{Cost}\left(V_{S O C, 1}, V_{S O C, 2}, i\right)$ from all the $\operatorname{Min} \_\operatorname{Cost}\left(V_{S O C, 1}^{\prime}, V_{S O C, 2}^{\prime}, i-1\right)$ values for $0 \leq V_{S O C, 1}^{\prime} \leq 100 \%$ and $0 \leq V_{S O C, 2}^{\prime} \leq 100 \%$ based on Observation II. We describe the calculation procedure in two steps as follows.

Step I (Calculation of battery array currents and availability check): The following equations calculate the lead acid battery array current $I_{L A}[i]$, which guarantees that the lead-acid battery array SoC changes from $V_{S O C, 1}^{\prime}$ to $V_{S O C, 1}$ in the time slot $i$ :

$$
\begin{aligned}
& I_{L A}[i] \cdot \eta_{\text {rate }, C, L A}\left(I_{L A}[i]\right) \cdot \Delta_{T}=\left(V_{S O C, 1}-V_{S O C, 1}^{\prime}\right) \cdot C_{f u l l, L A}, \\
& \text { if } V_{S O C, 1} \geq V_{S O C, 1}^{\prime}, \\
& I_{L A}[i] / \eta_{\text {rate }, d, L A}\left(\left|I_{L A}[i]\right|\right) \cdot \Delta_{T}=\left(V_{S O C, 1}-V_{S O C, 1}^{\prime}\right) \cdot C_{f u l l, L A}, \\
& \\
& \text { if } V_{S O C, 1}<V_{S O C, 1}^{\prime} .
\end{aligned}
$$

We calculate $I_{L I}[i]$ in a similar way, which guarantees that the Li-ion battery array SoC changes from $V_{S O C, 2}^{\prime}$ to $V_{S O C, 2}$ in the time slot $i$. However, we must make sure that $I_{L A}[i]$ and $I_{L I}[i]$ are within the range of the allowable charging/discharging current of the EES arrays. We check if constraints (15) or (16) are violated, i.e., $I_{L A}[i]$ is not in the range $\left[-I_{L A, M A X, d}, I_{L A, M A X, c}\right]$ or $I_{L I}[i]$ is not in the range $\left[-I_{L I, M A X, d}, I_{L I, M A X, c}\right]$. If constraints (15) or (16) are violated, we conclude that it is not possible for the lead-acid and Li-ion battery array SoCs to change simultaneously in the time slot $i$ from $V_{S O C, 1}^{\prime}$ to $V_{S O C, 1}$ and from $V_{S O C, 2}^{\prime}$ to $V_{S O C, 2}$, respectively. We proceed with another $\left(V_{S O C, 1}^{\prime}, V_{S O C, 2}^{\prime}\right)$ pair. If constraints (15) and (16) are not violated, we go on to the next step. This procedure is named availability check in Algorithm 1.

Step II (Calculation of $\left.\operatorname{Min} \_\operatorname{Cost}\left(V_{S O C, 1}, V_{S O C, 2}, i\right)\right)$ : First we calculate the optimal CTI voltage $V_{C T I}[i]$ that minimizes the energy cost in time slot $i$, or equivalently, maximizes $I_{\text {inv,grid }}[i]$, with given $I_{L A}[i]$ and $I_{L I}[i]$. Constraints in this optimization problem are the energy conservation constraints (8) - (12).

Maximization of $I_{\text {inv,grid }}[i]$ is in general a quasi-convex (unimodal) optimization problem over the variable $V_{C T I}[i]$. We exploit a ternary search algorithm, which is an extension of the wellknown binary search algorithm, utilizing this quasi-convex property. This makes the solution quickly converge to the global optimal or at least a near global optimal solution. A branch and bound method provides the global optimal solution of the optimization problem at the expense of the solution complexity. We calculate the minimal energy cost in time slot $i$ using Price $[i] \cdot V_{\text {grid }} \cdot\left(I_{\text {load }}[i]-\right.$ $\left.I_{\text {inv,grid }}[i]\right) \cdot \Delta_{T}$ after we find the maximum $I_{\text {inv,grid }}[i]$. We keep track of this minimal energy cost using $\operatorname{Min}_{-} \operatorname{Cost}_{i}\left(V_{S O C, 1}^{\prime}, V_{S O C, 2}^{\prime}, V_{S O C, 1}, V_{S O C, 2}\right)$.

We calculate Min_Cost $\left(V_{S O C, 1}, V_{S O C, 2}, i\right)$ using the following equation based on Observation II:

$$
\begin{aligned}
\operatorname{Min} \_\operatorname{Cost}\left(V_{S O C, 1}, V_{S O C, 2}, i\right)= \\
\min _{\left(V_{S O C, 1}^{\prime}, V_{S O C, 2}^{\prime}\right)} \operatorname{Min} \operatorname{Cost}\left(V_{S O C, 1}^{\prime}, V_{S O C, 2}^{\prime}, i-1\right)+ \\
\\
M i{ }_{1} \operatorname{Cost}_{i}\left(V_{S O C, 1}^{\prime}, V_{S O C, 2}^{\prime}, V_{S O C, 1}, V_{S O C, 2}\right) .
\end{aligned}
$$

We keep track of the optimal $V_{S O C, 1}^{\prime}$ and $V_{S O C, 2}^{\prime}$ using the following two matrices:

$$
\begin{aligned}
& \text { (Last_SoC } \left.{ }_{L A}\left(V_{S O C, 1}, V_{S O C, 2}, i\right) \text {, Last_SoC }{ }_{L I}\left(V_{S O C, 1}, V_{S O C, 2}, i\right)\right) \\
& =\arg \min _{\left(V_{S O C, 1}^{\prime}, V_{S O C, 2}^{\prime}\right)} \operatorname{Min} \_\operatorname{Cost}\left(V_{S O C, 1}^{\prime}, V_{S O C, 2}^{\prime}, i-1\right)+ \\
& \operatorname{Min}_{-} \operatorname{Cost}_{i}\left(V_{S O C, 1}^{\prime}, V_{S O C, 2}^{\prime}, V_{S O C, 1}, V_{S O C, 2}\right)
\end{aligned}
$$

which is necessary in finding the optimal control variable values after we find Min_Cost $\left(V_{S O C, 1}, V_{S O C, 2}, N\right)$.
After we calculate all the $\operatorname{Min} \_\operatorname{Cost}\left(V_{S O C, 1}, V_{S O C, 2}, N\right)$ values for $0 \leq V_{S O C, 1} \leq 100 \%$ and $0 \leq V_{S O C, 2} \leq 100 \%$, we determine the battery array SoCs $V_{S O C, L A}[N+1], V_{S O C, L I}[N+1]$ at the end of day using:

$$
\begin{aligned}
& \left(V_{S O C, L A}[N+1], V_{S O C, L I}[N+1]\right)= \\
& \underset{\arg \min }{\operatorname{Min}} \operatorname{Cost}\left(V_{S O C, 1}, V_{S O C, 2}, N\right) \text {. } \\
& \left(V_{S O C, 1} \geq V_{S O C, L A}[1], V_{S O C, 2} \geq V_{S O C, L I}[1]\right)
\end{aligned}
$$

This step is based on Observation I. We reflect the final stored energy constraints (17) in (22).

Next, we determine the optimal amounts of SoC change in both battery arrays in each time slot in a reverse chronological order. For example, the optimal amounts of SoC change in the lead-acid battery array and Li-ion battery array in time slot $N$ are $V_{S O C, L A}[N+1]-$ Last_SoC LA $\left._{S O C, L A}[N+1], V_{S O C, L I}[N+1], N\right)$ and $V_{S O C, L I}[N+$ $1]$ - Last_SoC $C_{L I}\left(V_{S O C, L A}[N+1], V_{S O C, L I}[N+1], N\right)$, respectively. We subsequently determine the optimal control variables values, i.e., the optimal $V_{C T I}[i], I_{L A}[i]$ and $I_{L I}[i]$ for $1 \leq i \leq N$. This process is called tracing back, and is the last step in dynamic programming [17]. Algorithm 1 discretizes the battery array SoC into $P$ levels. This discretization approach is necessary for effectively performing dynamic programming in the way of filling up the entries of a matrix. We exploit certain branch and bound techniques to reduce the computation time of Algorithm 1. However, such techniques are out of the scope of this paper.

\section{EXPERIMENTAL RESULTS}

We compare the proposed residential-level HEES system and its optimal control with baseline homogenous EES systems with control algorithm described in [16] in terms of the average profit. The proposed HEES system consists of a lead-acid battery array with 48 $\mathrm{V}$ nominal (average) terminal voltage and 30 Ahr nominal capacity, as well as a Li-ion battery array with $36 \mathrm{~V}$ nominal terminal voltage and 26.7 Ahr nominal capacity. The two baseline homogenous EES systems consist of either a lead-acid battery array or a Li-ion battery array. We obtain the lead-acid battery and Li-ion battery characteristics and parameters from real measurements. For fairness in comparison, we assume that the total battery array volumes in the proposed HEES system and in the two baseline systems are the same.

The residential electricity load and PV power generation profiles are measured at Duffield, in the year 2007. Figure 4 (a) shows a sample daily electrical load and PV power generation profiles while Figure 4 (b) shows the daily electricity energy price function adopted in the experiments.
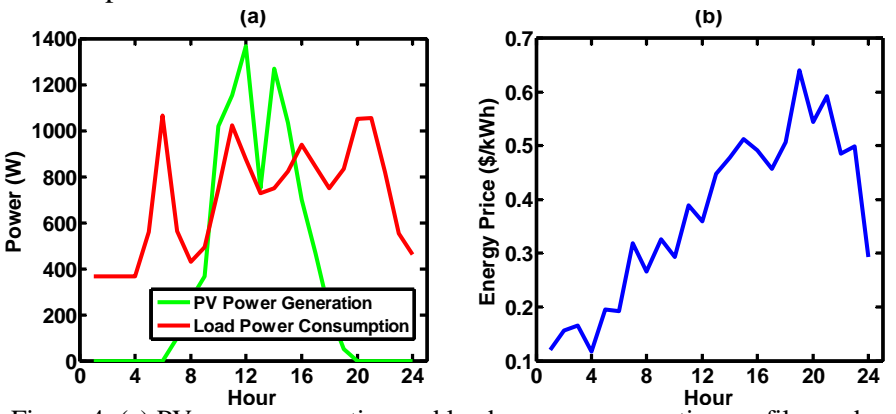

Figure 4: (a) PV power generation and load power consumption profiles and

(b) electricity energy price function.

We show the effectiveness of the proposed residential-level HEES system and its optimal control algorithm in Figure 5. Figure 5 provides the power profile drawn from the Grid without the HEES system where the load power consumption and PV power generation profiles are given in Figure 4 (a). It also provides the power profile 
drawn from the Grid when the proposed HEES system and the optimal HEES system control algorithm are incorporated. According to Figure 5, the HEES system could store power from the Grid and the PV system when the energy price is low, and perform peak shaving when the electricity price is high, thereby reducing the total energy cost over a billing period.

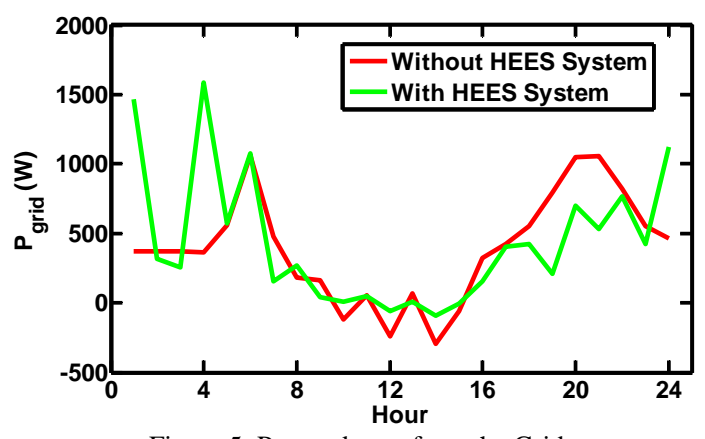

Figure 5: Power drawn from the Grid.

We compare the average profit enhancement between the proposed HEES system and baseline homogenous EES systems. We define the amortized cost of an EES array, or equivalently, a homogenous EES system, as the amortized daily capital cost required for purchasing and maintaining the EES array. For example, suppose that the capital cost of a Li-ion battery array is $\$ 3000$ and its life time is 5 years (1825 days.) The amortized cost is $\$ 3000 / 1825$ per day. The amortized cost of a HEES system is the sum of amortized costs of all its EES arrays. We define daily profit of the HEES (or baseline EES) system as the total electricity energy cost saving in a day due to the incorporation of the HEES (or baseline EES) system minus its amortized cost. We use $\$ 80 / \mathrm{kWh}$ and $\$ 350 / \mathrm{kWh}$ as the unit energy price of lead-acid battery and Li-ion battery, respectively [7]. Their lifetimes are given by 1.5 years and 5 years, respectively [7].

We show the daily profit of the proposed HEES system and the two baseline systems over a month of 30 days in Figure 6. The proposed HEES system outperforms both baseline systems in terms of the daily profit. It achieves an average daily profit enhancement of $73.9 \%$ compared with the baseline system consisting of only a leadacid battery bank due to its relatively low energy density and charging/discharging performance. The baseline system consisting of only a Li-ion battery bank even has a negative average daily profit due to its over-high capital cost. We conclude that the HEES system is a promising candidate compared to its homogeneous counterparts for residential Smart Grid usage.

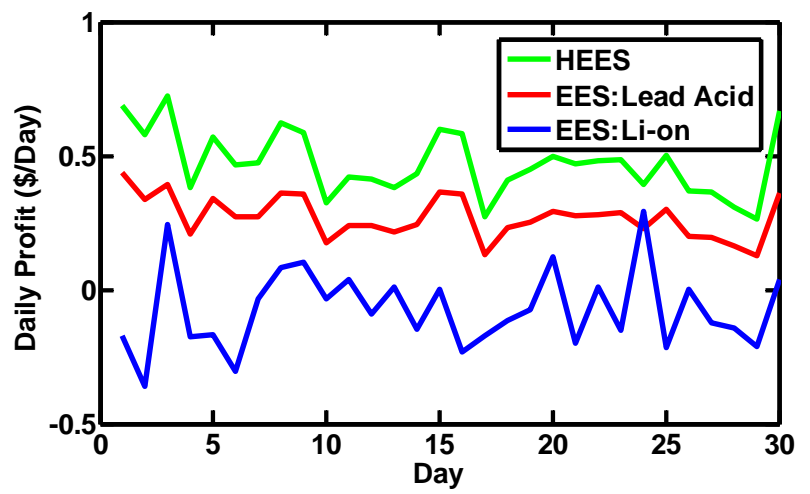

Figure 6: Daily profit of the proposed HEES system and two baseline systems.

\section{CONCLUSION}

This paper pioneers a problem of integrating the HEES system for a residential Smart Grid user equipped with PV power generation utility. We developed the optimal control algorithm for the residential-level HEES system. The objective of the optimal control algorithm is to reduce the total electricity cost over a billing period (i.e., a day) and perform peak power shaving under arbitrary energy price function. The proposed optimal HEES system control algorithm correctly accounts for the distinct characteristics of different types of EES elements, conversion efficiency variations of power converters, as well as the TOU (time-of-use) dependent energy price function. The proposed algorithm is based on dynamic programming and therefore has polynomial time complexity. Experimental results demonstrate that the proposed algorithm achieves up to $73.9 \%$ average profit enhancement compared with baseline EES systems.

\section{REFERENCES}

[1] L. D. Kannberg et al., "GridWise ${ }^{\mathrm{TM}}$ : The benefits of a transformed energy system,” PNNL-14396, Pacific Northwest National Laboratory, Richland, Sep. 2003.

[2] S. Kishore and L. V. Snyder, "Control mechanisms for residential electricity demand in SmartGrids," Proc. of Smart Grid Communications (SmartGridComm) Conference, 2010.

[3] S. Caron and G. Kesidis, "Incentive-based energy consumption scheduling algorithms for the Smart Grid," Proc. of Smart Grid Communications (SmartGridComm) Conference, 2010.

[4] H. T. Odum, "Energy quality and carrying capacity of the earth,” Tropical Ecology, 1975.

[5] J. Baker and A. Collinson, "Electrical energy storage at the turn of the millennium,” Power Engineering Journal, 1999.

[6] T. Moore and J. Douglas, "Energy storage, big opportunities on a smaller scale,” EPRI J., 2006.

[7] M. Pedram, N. Chang, Y. Kim, and Y. Wang, "Hybrid electrical energy storage systems," in ISLPED, 2010.

[8] F. Koushanfar, "Hierarchical hybrid power supply networks," in DAC, 2010.

[9] Y. Wang, Y. Kim, Q. Xie, N. Chang, and M. Pedram, “ Charge migration efficiency optimization in hybrid electrical energy storage (HEES) systems,” in ISLPED, 2011.

[10] Q. Xie, Y. Wang, Y. Kim, N. Chang, and M. Pedram, "Charge allocation for hybrid electrical energy storage systems," in CODES+ISSS, 2012.

[11] Q. Xie, Y. Wang, M. Pedram, Y. Kim, D. Shin, and N. Chang, "Charge replacement in hybrid electrical energy storage systems," in ASP-DAC, 2012.

[12] M. Chen and G. Rincon-Mora, "Accurate electrical battery model capable of predicting runtime and I-V performance," IEEE T. on Energy Conversion, 2006.

[13] D. Shin, Y. Wang, Y. Kim, J. Seo, M. Pedram, and N. Chang, "Battery-supercapacitor hybrid system for high-rate pulsed load applications," in DATE, 2011.

[14] D. Linden and T. B. Reddy, Handbook of Batteries. McGrawHill Professional, 2001.

[15] T. Esram, J. Kimball, P. Krein, P. Chapman, and P. Midya, "Dynamic maximum power point tracking of photovoltaic arrays using ripple correlation control," IEEE Trans. on Power Electronics, 2006.

[16] S. Park, Y. Wang, Y. Kim, N. Chang, and M. Pedram, "Battery management for grid-connected PV systems with a battery," in ISLPED 2012.

[17] T. H. Cormen, C. E. Leiserson, R. L. Rivest, and C. Stein, Introduction to Algorithms ( ${ }^{\text {rd }}$ ed.). MIT Press and McGraw Hill, 2009. 